Chirurg 2014 $\cdot 85: 642$

DOI 10.1007/s00104-014-2791-1

Online publiziert: 10. Juli 2014

๑) Springer-Verlag Berlin Heidelberg 2014

J. Reibetanz · C.T. Germer

Klinik für Allgemein-, Viszeral-, Gefäß- und Kinderchirurgie, Universitätsklinik Würzburg

\title{
Einfluss der strukturierten CEA-Bestimmung und CT-Diagnostik auf die Rezidivdiagnose beim kolorektalen Karzinom
}

4. „minimum follow-up: nur einmalige CT-Untersuchung zwischen 12 und 18 Monaten nach Primärtherapie.

Primrose JN et al (2014) Effect of 3 to 5 years of scheduled CEA and CT follow-up to detect recurrence of colorectal cancer: the FACS randomized clinical trial. JAMA 311:263-270

\section{Hintergrund}

Eine strukturierte Nachsorge soll beim kolorektalen Karzinom (CRC) im Stadium II und III zur frühzeitigen Diagnose eines (potenziell kurativ therapierbaren) Rezidivs durchgeführt werden. Hieraus lässt sich ein Überlebensvorteil für intensiver nachgesorgte Patienten nach 5 Jahren ableiten [1].

\section{Fragestellung der Studie}

Ziel der prospektiv-randomisierten FACS (follow-up after colorectal cancer surgery) Studie war die Überprüfung der Effektivität einer planmäßigen CEA (karzinoembryonales Antigen) -Bestimmung und/ oder Computertomographie (CT) gegenüber einem "minimum follow-up“ in der Diagnose eines Tumorrezidivs, das einer kurativen Therapie zugänglich ist. Hierzu wurden in der Zeit zwischen Januar 2003 und August 2009 insgesamt 1202 Patienten nach kurativer Therapie eines CRC aus 39 britischen Zentren rekrutiert und in eine der folgenden 4 Gruppen randomisiert:

1. CEA-Bestimmung alle 3 Monate innerhalb der ersten 2 Jahre, dann halbjährlich bis zum 5. Jahr $(\mathrm{n}=300)$;

2. CT-Thorax-Abdomen-Becken alle 6 Monate innerhalb der ersten 2 Jahre, dann jährlich bis zum 5. Jahr $(n=299)$;

3. CEA-Bestimmung und CT zu o. g. Intervallen $(\mathrm{n}=302)$;
Primärer Endpunkt war die kurativ-intendierte chirurgische Therapie eines Tumorrezidivs nach einem minimalen Follow-up von 3 Jahren. Sekundäre Endpunkte waren tumorbedingtes Versterben, Diagnosezeitpunkt des Rezidivs und Überleben nach Therapie des Rezidivs.

\section{Ergebnisse}

Während des mittleren Nachsorgeintervalls von 4,4 Jahren $( \pm 0,8)$ wurden insgesamt 199 Rezidivfälle diagnostiziert $(16,6 \%)$, davon $41(3,4 \%)$ ausschließlich lokoregionäre und $101(8,4 \%)$ metastatische Rezidive in Lunge und/oder Leber. Innerhalb der 3 Interventionsgruppen mit planmäßiger CEA- und/oder CT-Untersuchung wurde das Tumorrezidiv tendenziell früher diagnostiziert $(\mathrm{p}=0,18)$ als in der „Minimum-follow-up“-Gruppe. Ein kurativ-intendierter chirurgischer Eingriff zur Therapie des Tumorrezidivs kam in der „Minimum-follow-up“-Gruppe in 2,3\% der Fälle zur Anwendung und damit signifikant seltener als in jeder der Interventionsgruppen: in der CEA-Gruppe 4,4\% [95\%-Konfidenzintervall (KI) 1,07,9\%, Odds Ratio: 3,0], in der CEA+CTGruppe 4,3\% [95\%-KI 1,0-7,9\%, Odds Ratio: 3,1] und in der CT-Gruppe 5,7\% [95\%-KI 2,2-9,5\%, Odds Ratio: 3,6]. Das Überleben unterschied sich zwischen den einzelnen Gruppen nicht signifikant.

\section{Diskussion und Fazit des Reviewers}

Im Kapitel Nachsorge empfiehlt die aktuelle Leitlinie „Kolorektales Karzinom“ im Hinblick auf Tumormarker und CTUntersuchung (lediglich) eine 6-monatliche CEA-Bestimmung für mindestens 2 Jahre und sieht keine Indikation zum routinemäßigen Einsatz der CT in der Nachsorge [2]. Die vorgelegte Studie zeigt jedoch eindrücklich, dass Patienten nach kurativer Primärtherapie eines kolorektalen Karzinoms von einem intensiveren Follow-up mittels standardisierter CEABestimmung alle 3 Monate oder einer routinemäßigen CT-Untersuchung hinsichtlich der (frühzeitigen) Diagnose eines potenziell kurativ therapierbaren Tumorrezidivs profitieren (wobei ein weiterer Vorteil durch die Kombination beider Modalitäten jedoch nicht besteht). Eine Intensivierung der Tumornachsorge über die Leitlinienempfehlung hinaus scheint somit im Interesse des Patienten.

\section{Korrespondenzadresse}

\section{Prof. Dr. C.T. Germer}

Klinik für Allgemein-, Viszeral-, Gefäß- und Kinderchirurgie, Universitätsklinik Würzburg, Oberdürrbacher Str. 6, 97080 Würzburg germer_c@ukw.de

\section{Einhaltung ethischer Richtlinien}

Interessenkonflikt. J. Reibetanz und C.T. Germer geben an, dass kein Interessenkonflikt besteht.

\section{Literatur}

1. Jeffery M, Hickey BE, Hider PN (2007) Follow-up strategies for patients treated for non-metastatic colorectal cancer. Cochrane Database Syst Rev 1:CD002200

2. Pox C, Aretz S, Bischoff SC et al (2013) S3-Leitlinie Kolorektales Karzinom. Z Gastroenterol 51:753854 